\title{
Endogenous Myc maintains the tumor microenvironment
}

\author{
Nicole M. Sodir, ${ }^{1,2}$ Lamorna Brown Swigart, ${ }^{1,2}$ Anthony N. Karnezis, ${ }^{1,2}$ Douglas Hanahan, ${ }^{3}$ \\ Gerard I. Evan, ${ }^{1,2,4,6}$ and Laura Soucek ${ }^{1,2,5}$ \\ ${ }^{1}$ Department of Pathology, University of California at San Francisco, San Francisco, California 94143, USA; ${ }^{2}$ Helen Diller Family \\ Comprehensive Cancer Center, University of California at San Francisco, San Francisco, California 94143, USA; ${ }^{3}$ Swiss Institute \\ for Experimental Cancer Research, Ecole Polytechnique Federale Lausanne, The Swiss Federal Institute of Technology, Lausanne \\ CH-1015, Switzerland; ${ }^{4}$ Department of Biochemistry, University of Cambridge, Cambridge CB2 1GA, United Kingdom
}

\begin{abstract}
The ubiquitous deregulation of Myc in human cancers makes it an intriguing therapeutic target, a notion supported by recent studies in Ras-driven lung tumors showing that inhibiting endogenous Myc triggers ubiquitous tumor regression. However, neither the therapeutic mechanism nor the applicability of Myc inhibition to other tumor types driven by other oncogenic mechanisms is established. Here, we show that inhibition of endogenous Myc also triggers ubiquitous regression of tumors in a simian virus 40 (SV40)-driven pancreatic islet tumor model. Such regression is presaged by collapse of the tumor microenvironment and involution of tumor vasculature. Hence, in addition to its diverse intracellular roles, endogenous Myc serves an essential and nonredundant role in coupling diverse intracellular oncogenic pathways to the tumor microenvironment, further bolstering its credentials as a pharmacological target.
\end{abstract}

[Keywords: Myc inhibition; pancreas; tumor; microenvironment; therapeutics]

Supplemental material is available for this article.

Received February 2, 2011; revised version accepted March 15, 2011.

Tumors are dysfunctional tissues wherein cancer cells reside in an interdependent association with a somatic microenvironment comprised of diverse stromal, vascular, and inflammatory cell types. Evidence has amassed that the various normal somatic cells that make up the tumor microenvironment are essential for various aspects of macroscopic tumor growth, maintenance, invasion, and angiogenesis, and profoundly influence initial responses to therapy, tumor recurrence, and the evolution of drug resistance. Unfortunately, in established tumors, cancer cells and their adjacent stroma are so reciprocally intertwined that the cause-and-effect relationships linking them have proven very difficult to disentangle: It remains unclear to what extent tumor cells instruct their microenvironment versus the microenvironment instructs the tumor cells, or the extent to which each is dependent on the other for its maintenance.

Nonetheless, the obligate role in tumor growth and maintenance played by the tumor microenvironment has prompted a variety of novel therapeutic approaches aimed at neutralizing tumor cell-nonautonomous components of

${ }^{5}$ Present address: Vall d'Hebron Institute of Oncology (V.H.I.O), Barcelona 08035, Spain.

${ }^{6}$ Corresponding author.

E-MAIL gie20@cam.ac.uk; FAX (415) 514-0878

Article published online ahead of print. Article and publication date are online at http://www.genesdev.org/cgi/doi/10.1101/gad.2038411. the tumor mass, most notably tumor vasculature and inflammation. These approaches seek to capitalize on the attractive notion that, by aiming at the normal stromal compartment of tumors rather than genetically unstable cancer clones, evolution of drug resistance should be greatly reduced. Unfortunately, by and large, such therapies have not proven especially effective. In great part, this is because the extracellular programs that craft and maintain tissues are inherently degenerate and robust, as befits processes whose imperative is to build and maintain consistent tissue architectures even when the precise signals driving such processes are both varied and variable (Coussens et al. 2002; Fingleton 2003; Miller et al. 2005; Bergers and Hanahan 2008). Effective and durable therapies aimed at the tumor microenvironment will require the identification and specific targeting of pathway effectors that are both nondegenerate and obligate.

Myc is a basic helix-loop-helix leucine zipper transcription factor whose activity is deregulated in most human cancers. Acute ectopic activation of Myc is sufficient in many tissues to engage and maintain the diverse intracellular programs (cell growth, cell cycle progression, biosynthetic metabolism, ribogenesis, and translation) (Meyer and Penn 2008) and extracellular programs (release of cytokines and chemokines, recruitment of inflammatory cells, extensive stromal remodeling, invasion, and angiogenesis) that normal and neoplastic somatic cells need for their expansion 
(Pelengaris et al. 1999, 2002; Shchors et al. 2006; Soucek et al. 2007). This pleiotropic role hints that Myc inhibition might be an especially efficacious therapeutic strategy, since it could concomitantly incapacitate both intracellular and extracellular oncogenic pathways. Consistent with this, we recently used a dominant-negative approach to inhibit Myc systemically in vivo to show that endogenous Myc is essential for maintenance of oncogenic KRasinduced tumors of the lung (Soucek et al. 2008). However, key questions remain: Most notably, is the dependence on Myc for tumor maintenance a tumor cell-autonomous or tumor cell-nonautonomous process, and how general is the requirement for Myc in other tumor types driven in other tissues by other oncogenic mechanisms?

The well-characterized rat insulin promoter 1 (RIP1)Tag2 mouse model of multistage pancreatic $\beta$-cell carcinogenesis is a workhorse for studying how the microenvironment modulates tumor growth (Christofori and Hanahan 1994; Bergers and Hanahan 2008). RIP1-Tag2 tumorigenesis is driven by transgenic expression of simian virus 40 (SV40) large $\mathrm{T}$ and small $\mathrm{t}$ antigens from the RIP (Hanahan 1985). All RIP1-Tag2 animals develop hyperplastic/dysplastic islets at $\sim 5-7$ wk of age (Hanahan 1985 ), and, at $\sim 9$ wk of age, some of these lesions transition to a highly angiogenic phenotype that exhibits histological features of high-grade dysplasia (Folkman et al. 1989). Thereafter, a subset of these angiogenic lesions evolves into solid tumors. RIP1-Tag2 mice typically die from tumor burden and associated hyperinsulinemia at $\sim 14 \mathrm{wk}$ (Christofori and Hanahan 1994). The protracted, stepwise, and sporadic kinetics of tumor evolution in RIP1-Tag2 mice intimate that SV40 T/t antigens are insufficient to pilot tumorigenesis through various evolutionary bottlenecks without the cooperation of additional mutations. Furthermore, at least some of these crucial bottlenecks are thought to reflect inadequacies in the capacity of the normal somatic microenvironment to support vigorous tumor expansion. Most notable of these is the angiogenic switch, a complex and functionally degenerate tissue transition involving recruitment of inflammatory cells, release of proteolytic and angiogenic effector molecules, extensive stromal remodeling, and vascular elaboration. The root cause of this dramatic shift in tissue dynamics and local microenvironment remains unknown; in particular, to what extent is tumor angiogenesis dependent on tumor cell-specific signals as opposed to tumor cell-extrinsic signals that originate within the host stroma?

To address these questions directly, we combined the RIP1-Tag2 pancreatic $\beta$-cell mouse tumor model with our TRE-Omomyc;CMVrtTA mouse, in which the dominantnegative Myc inhibitor Omomyc (Soucek et al. 1998, 2002, 2004) may be reversibly induced systemically in vivo (Soucek et al. 2008). Omomyc competitively blocks Myc/Max heterodimerization and binding to the E-box, thus inhibiting the capacity of Myc proteins to transactivate target genes (Soucek et al. 1998, 2002). Tumorigenesis in the RIP1-Tag2 model is driven by a completely different oncogenic mechanism from the KRas ${ }^{G 12 D}$ lung tumor model, in a very different tissue type in which the tumor microenvironment plays a critical part in tumor progression and maintenance. We used this model to establish the role that endogenous Myc within tumor cells plays in maintenance of $\beta$-cell tumors and their peculiar microenvironment.

\section{Results}

Endogenous Myc is required for progression and maintenance of RIP1-Tag2 islet tumors

Expression of the dominant inhibitory Myc dimerization domain mutant Omomyc in TRE-Omomyc;CMVrtTA mice is driven from a tetracycline-responsive promoter element (TRE) whose activity is reliant on a reverse tetracycline-dependent transactivator, rtTA (Tet-On system), expressed off the $C M V$ promoter (Soucek et al. 2008). The $C M V$ promoter is highly active in most adult mouse tissue types (Furth et al. 1991; Kothary et al. 1991; Baskar et al. 1996; Soucek et al. 2008), and administration of doxycycline to TRE-Omomyc;CMVrtTA mice elicits widespread inhibition of endogenous Myc trans-activation, including exocrine and endocrine pancreas (Soucek et al. 2008). TRE-Omomyc;CMVrtTA mice were crossed into the RIP1-Tag2 mouse model of pancreatic $\beta$-cell cancer (Hanahan 1985). In the absence of doxycycline, TREOmomyc;CMVrtTA;RIP1-Tag2 triple-transgenic mice developed tumors with incidence, multiplicity, and kinetics of tumor progression indistinguishable from their RIP1Tag2 littermates (Supplemental Fig. 1). All of the tumors examined were well or moderately differentiated neuroendocrine tumors, based on clinical and pathological criteria, and appeared to be circumscribed, nodular masses with noninfiltrative borders (Fig. 1).

To ascertain the role of endogenous Myc function in the pathogenesis of SV40 T/t-driven islet cancer in RIP1-Tag2 mice, we first asked whether inhibition of Myc prevents expansion and progression of RIP1-Tag2-driven tumors. Systemic Omomyc expression was induced in 7-wk-old TRE-Omomyc;CMVrtTA;RIP1-Tag2 mice by addition of doxycycline to their drinking water for seven subsequent weeks, then the animals were sacrificed and their pancreata were harvested and assessed immunohistochemically. As expected, at $14 \mathrm{wk}$ of age, RIP1-Tag2 mice exhibited the predicted distribution of islet tumor sizes and grades as described in Christofori and Hanahan (1994). In contrast, no overt islet tumors were present in Omomycexpressing animals (TRE-Omomyc;CMVrtTA;RIP1-Tag2 mice treated with doxycycline). Indeed, both islet size and distribution remained similar to that of control littermates not expressing RIP1-Tag2 (Fig. 1A; lowpower-magnification images are also provided as Supplemental Fig. 2). Hence, Myc inhibition prevents formation of RIP1-Tag2 $\beta$-cell tumors.

To assess the impact of inhibiting endogenous Myc on established RIP1-Tag2 islet tumors, we induced Omomyc expression in 11-wk-old TRE-Omomyc;CMVrtTA;RIP1Tag2 mice-which at this age harbor hyperplastic lesions, angiogenic tumors, and carcinomas (Supplemental Fig. 3) - and maintained Omomyc expression for three 

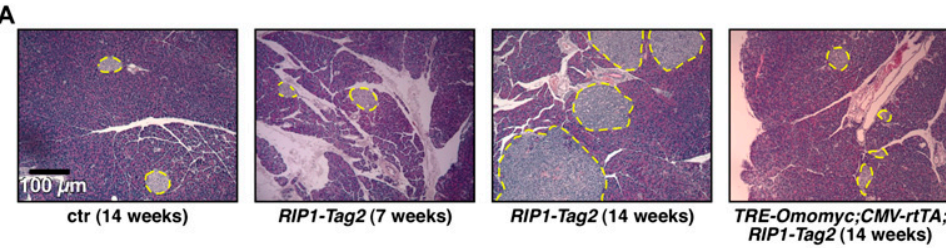

B

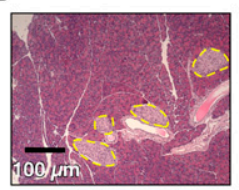

ctr (14 weeks)

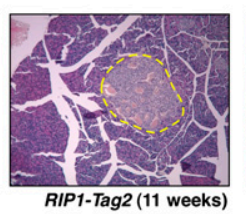

RIP1-Tag2 (11 weeks)
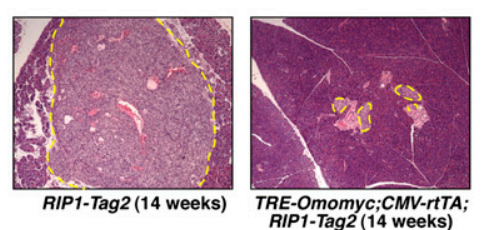

Figure 1. Endogenous Myc function is required for progression and maintenance of RIP1-Tag2-induced tumors. (A) Hematoxylin and eosin (H\&E) staining of pancreata sections from 14-wk-old nontransgenic controls (ctr), 7-wk-old RIP1-Tag2, 14-wk-old RIP1-Tag2, and 14-wk-old TRE-Omomyc;CMVrtTA;RIP1-Tag2 mice treated with doxycycline for 7 wk prior to sacrifice. Yellow broken lines delineate islet/tumor borders. Ages of mice at sacrifice are shown in brackets. $(B) \mathrm{H} \& \mathrm{E}$ staining of pancreata sections from 14-wk-old nontransgenic controls (ctr), 11-wk-old RIP1-Tag2, 14-wk-old untreated TRE-Omomyc;CMVrtTA;RIP1-Tag2 mice, and 14-wk-old TRE-Omomyc;CMVrtTA;RIP1-Tag2 mice treated with doxycycline for 3 wk prior to sacrifice. Yellow broken lines delineate islet borders. Ages of mice at sacrifice are shown in brackets (see also Supplemental Figs. 1-4).

further weeks. Pancreata were then harvested, and tumor status was assessed histologically. Strikingly, whereas the 14-wk-old RIP1-Tag2 Omomyc-negative control group exhibited multiple, large, angiogenic, and aggressive islet tumors, Omomyc-expressing animals (TRE-Omomyc; CMVrtTA;RIP1-Tag2 mice treated with doxycycline) lacked any such lesions (Fig. 1B; low-power-magnification images are also provided as Supplemental Fig. 2). To exclude the formal possibility that Omomyc suppresses expression of SV40 T antigens driven from the insulin promoter in RIP1Tag2 islets, we directly assayed expression of SV40 large $\mathrm{T}$ immunohistochemically. Omomyc induction had no impact on T-antigen expression in RIP1-Tag2 $\beta$ cells (Supplemental Fig. 4).

To investigate the mechanism of islet tumor regression in RIP1-Tag2 mice following Myc inhibition, we induced Omomyc expression for $1 \mathrm{wk}$ in 13-wk-old TRE-Omomyc; CMVrtTA;RIP1-Tag2 mice and assayed both proliferation and apoptosis. Although overall tumor size is not significantly reduced after only 1 wk of Omomyc expression, Omomyc expression elicited both a profound suppression of tumor cell proliferation and a significant increase in apoptosis (Fig. 2A), irrespective of tumor grade or stage (Supplemental Fig. 5). Hence, Myc inhibition is both cytostatic and cytotoxic in RIP1-Tag2 tumors. Nonetheless, the $\beta$ cells in the regressed islet tumors of Omomycexpressing TRE-Omomyc;CMVrtTA;RIP1-Tag2 mice retained the classic aberrant cytologic features of transformed $\beta$ cells (Fig. 2B), indicating that Myc inhibition does not block SV40 T/t-oncogenic function per se, only the propagation and expansion of such transformed cells. Of note, the regressed islet tumors retained strong peripheral glucagon expression, and, in general, the ratio of glucagon-positive $\alpha$ cells to insulin-positive $\beta$ cells in Omomyc-regressed tumors was comparable with that in normal islets (Fig. 2C).

\section{Endogenous $M y c$ is required for maintenance} of the RIP1-Tag2 islet tumor microenvironment

Inactivation of ectopic Myc in Myc-driven $\beta$-cell tumors triggers rapid degeneration of the tumor microenviron- ment, marked by collapse of tumor vasculature (Pelengaris et al. 2002; Shchors et al. 2006; Soucek et al. 2007). However, whether endogenous Myc plays an analogous role in driving and maintaining the microenvironment in tumors driven by other oncogenes is unknown. Since the switch to an angiogenic phenotype is a prerequisite for expansion, invasion, and metastasis of RIP1-Tag2 tumors (Bergers et al. 2000), we next examined the impact of Myc inhibition on the microenvironment supporting established RIP1-Tag2 islet tumors. First, Omomyc was induced in 13-wk-old RIP1-Tag2 mice for $1 \mathrm{wk}$, and tumor hypoxia was assessed. Hypoxia was absent from all normal islets in control mice and was only occasionally evident in some regions of the many large (but extensively vascularized) $\beta$-cell tumors of RIP1-Tag2 mice ( $19 \% \pm 3.8 \%$ of tumors). In contrast, hypoxia was widespread among islet tumors from Omomyc-expressing TRE-Omomyc;CMVrtTA;RIP1Tag2 mice $(68.75 \% \pm 9.5 \%$ of tumors) (Fig. 3A). Such regions of hypoxia coincided with regions of significant endothelial cell death $127 \% \pm 3.2 \%$ of all dying cells in angiogenic lesions and overt tumors of Omomyc-expressing TRE-Omomyc;CMVrtTA;RIP1-Tag2 mice are endothelial), suggesting that hypoxia arises from attrition of tumor vasculature (Fig. 3B). Detailed kinetic analysis of such phenomenon by double staining for Meca32 and active Caspase 3 clearly showed that, at $3 \mathrm{~d}$ after doxycycline treatment, the great majority of apoptotic cells in the TREOmomyc;CMVrtTA;RIP1-Tag2 mice are endothelial, not tumor (Fig. 4). Onset of tumor cell death follows rapidly thereafter, and, by $7 \mathrm{~d}$ of Myc inhibition, the majority of cells dying are tumor $\beta$ cells. Hence, vasculature collapse precedes tumor death and is not a secondary consequence of tumor regression. Concomitant analysis of pericytes by immunohistochemistry with NG2 antibody revealed only occasional cell deaths at either 3 or $7 \mathrm{~d}$ of Myc inhibition (Supplemental Fig. 6).

The principle proximal mediator of angiogenesis in RIP1-Tag2 tumors is VEGF, which is constitutively present in normal pancreatic islets but is sequestered in an inactive state by the extracellular matrix. The onset of islet tumor angiogenesis in RIP1-Tag2 mice is precipitated by proteolytic release of ECM-bound VEGF and 
Sodir et al.

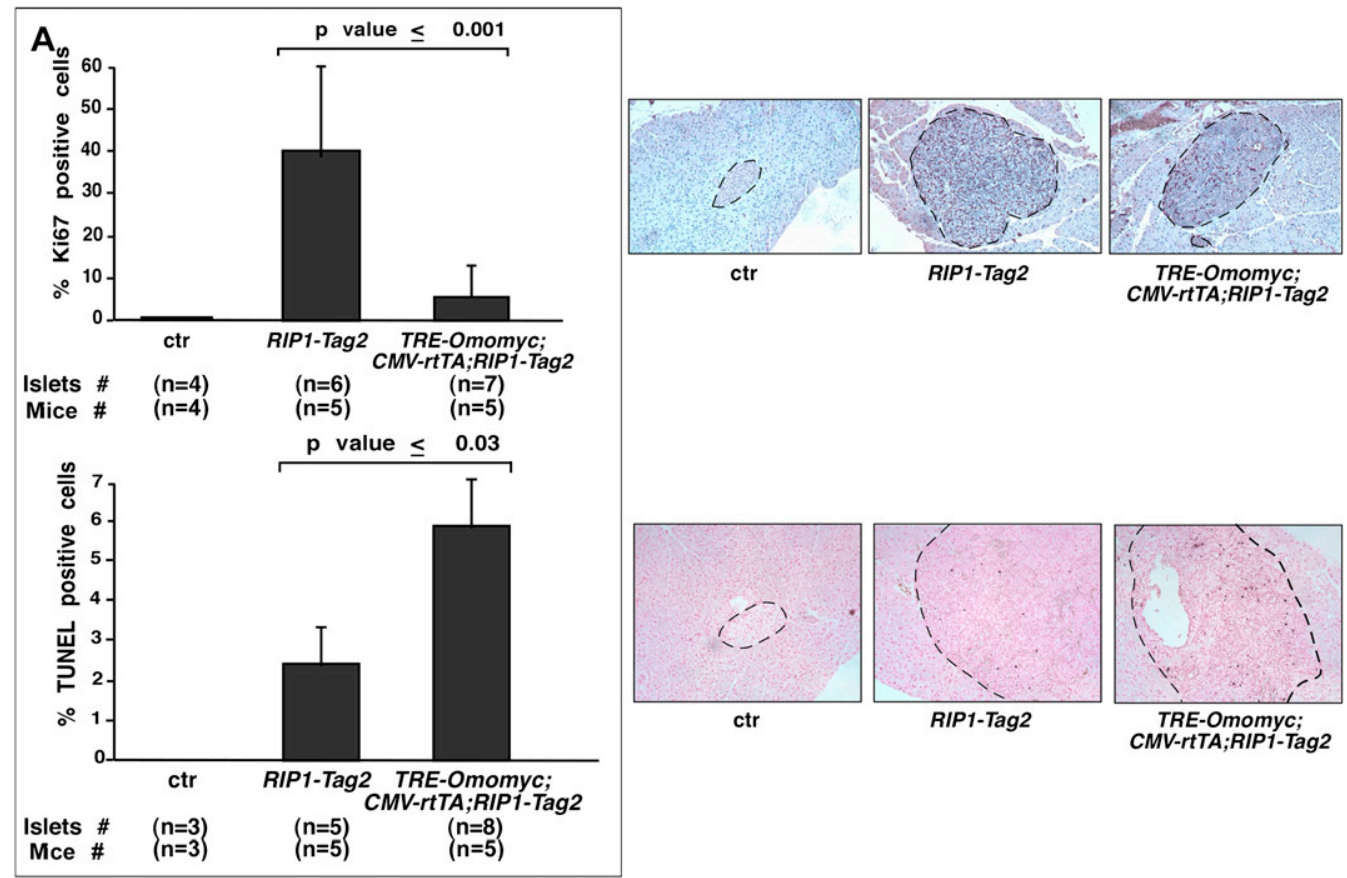

B

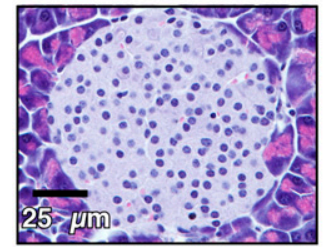

ctr (14 weeks)

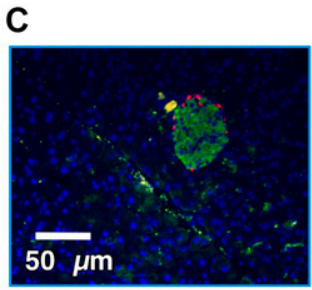

ctr (14 weeks)

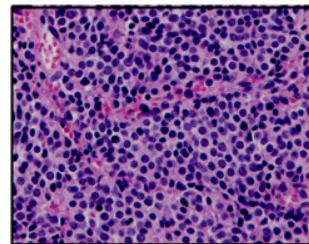

RIP1-Tag2 (14 weeks)

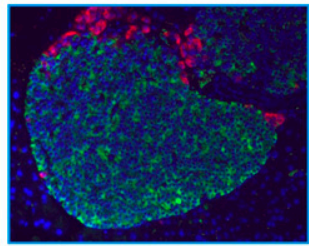

RIP1-Tag2

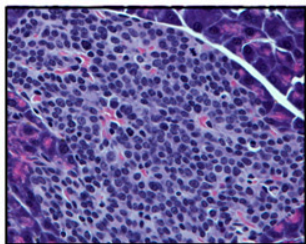

TRE-Omomyc;CMV-rtTA; RIP1-Tag2 (14 weeks)

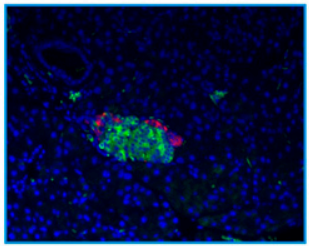

TRE-Omomyc;CMV-rtTA; RIP1-Tag2 (14 weeks)

Figure 2. Inhibition of endogenous Myc function decreases proliferation and increases apoptosis in RIP1-Tag2 pancreatic $\beta$ cells but does not suppress SV40 T/t-induced cellular transformation or disrupt $\alpha$-cell disposition. $(A)$ Immunohistochemical quantitation of Ki67-positive (top) and TUNEL-positive (bottom) cells in islets from 14-wk-old nontransgenic control (ctr), RIP1-Tag2, and TREOmomyc;CMVrtTA;RIP1-Tag2 mice treated with doxycycline for 1 wk prior to sacrifice. Data are represented as mean + SD. Representative images of islet/ $\beta$-cell tumors used in this immunohistochemical quantitation are shown on the right (see also Supplemental Fig. 5). (B) Higher-magnification images of H\&E-stained islets isolated from 14-wk-old nontransgenic control (ctr), RIP1Tag2, and TRE-Omomyc;CMVrtTA;RIP1-Tag2 mice treated with doxycycline for 3 wk prior to sacrifice showing aberrant transformed morphology of $\beta$ cells in RIP1-Tag2 and TRE-Omomyc;CMVrtTA;RIP1-Tag2 $\beta$-cell tumors. (C) Immunohistochemical staining of glucagon-expressing $\alpha$ cells (in red) and insulin-expressing $\beta$ cells (in green) in islets from 14-wk-old nontransgenic control (ctr), RIP1Tag2, and TRE-Omomyc;CMVrtTA;RIP1-Tag2 mice treated with doxycycline for 3 wk prior to sacrifice. Nuclear Hoechst counterstaining is shown in blue.

ligation to its principal receptor, VEGF-R2/Flk-1 (Bergers et al. 2000). Since Myc inhibition triggers rapid vascular attrition, we ascertained whether Myc inhibition abrogates VEGF:VEGF-R2 ligation by staining pancreas sections from Omomyc-expressing TRE-Omomyc;CMVrtTA;
RIP1-Tag2 mice with the GVM39 monoclonal antibody that specifically recognizes VEGF:VEGFR2 (Flk1)-ligated conjugates (Brekken et al. 1998). Omomyc expression blocked all detectable interaction between VEGF and its receptor (Fig. 5), offering a compelling proximal mechanism 
A
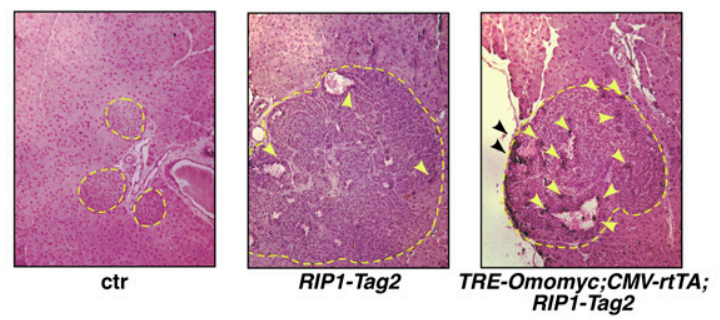

B

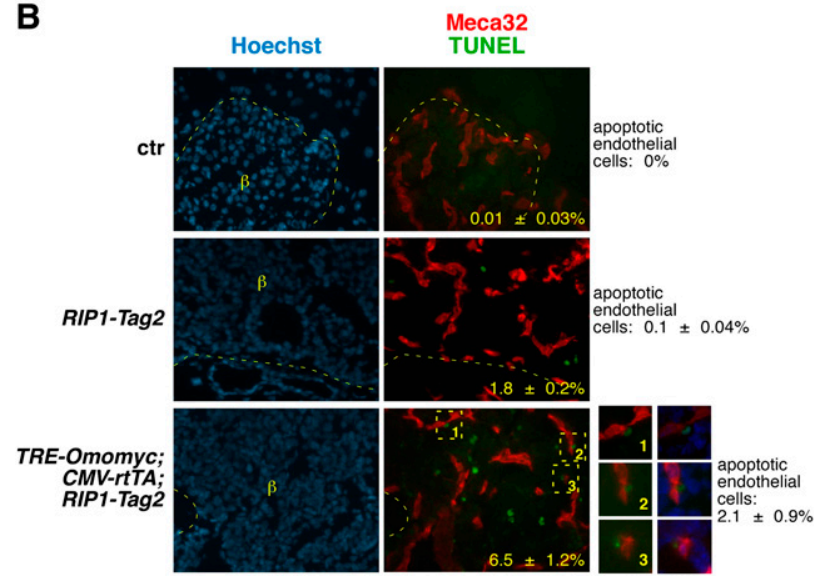

Figure 3. Endogenous $\mathrm{Myc}$ is required for maintenance of RIP1-Tag2 tumor vasculature. (A) Immunostaining with hypoxyprobe-1 antibody (darker areas indicated by arrowheads indicate hypoxic regions superimposed on Fast Red Nuclear counterstain) in sections of pancreata harvested from 14-wk-old nontransgenic control (ctr) mice (left), RIP1-Tag2 mice (middle), and TRE-Omomyc;CMVrtTA;RIP1-Tag2 mice (right) treated with doxycycline for $1 \mathrm{wk}$ prior to sacrifice. Broken lines delineate tumor borders. (B) Immunostaining for the endothelial cell marker Meca32 (in red) and apoptosis (TUNEL in green) of sections from pancreata harvested from the same mice as in A. Insets $i-$ iii show representative higher-magnification images of TUNEL-positive apoptotic endothelial cells (see also Supplemental Fig. 6).

for attrition of islet tumor vasculature following Myc inhibition.

The immediate proximal trigger for release of ECMbound VEGF that drives and maintains RIP1-Tag2 islet tumor angiogenesis is activation of matrix metalloprotease type 9 (MMP9) (Bergers et al. 2000). MMP9 is produced by two principal inflammatory cell types in RIP1-Tag2 islets (macrophages and neutrophils) (Nozawa et al. 2006) that home to incipient tumors in response to release of various chemoattractants (Shojaei et al. 2008). Given their crucial role in the angiogenic step of RIP1-Tag2 islets, we ascertained whether both the location and number of these inflammatory cells are modulated by Myc inhibition. We verified that macrophages accumulate along the peripheries of RIP1-Tag2 tumors, where they are thought to orchestrate interactions with surrounding stroma, while neutrophils accumulate in the tumor interior, as described by Nozawa et al. (2006) (Fig. 6). In contrast, both macrophages and neutrophils were completely absent from tumors of TRE-Omomyc;CMVrtTA;RIP1-Tag2 mice expressing Omomyc for only $3 \mathrm{~d}$ (Fig. 6), indicating that sustained expression of endogenous Myc is required for both recruitment and retention of inflammatory cells by established RIP1-Tag2 tumors.

To identify possible mediators of the Myc-dependent cross-talk between tumor and microenvironment, we used an unbiased approach to identify inflammatory and vascular modulators that are directly and rapidly influenced by Myc inhibition. Laser-captured tumor islets were isolated from the pancreata of 13-wk-old TRE-Omomyc; CMVrtTA;RIP1-Tag2 mice treated with either doxycyline or sucrose for $2 \mathrm{~d}$, and gene expression was profiled and compared. Our analysis focused on cytokine, chemokine, and inflammatory response genes described in the PCR arrays from SAbiosciences (http://www.sabiosciences. com/Cytokines_Inflammation.php). We then identified only those genes that showed a fold change of at least 0.2 between Omomyc-treated and untreated states. We found that multiple genes implicated in inflammation and inflammocyte recruitment were rapidly modulated by Myc inhibition (Fig. 7). Some of the genes down-regulated upon Myc inhibition-such as IL8rb, Il19, Il3, LTb4r2, Il11, Ccl7, Ccl8, and Ccl12-have well-characterized roles in chemotaxis, proliferation, and maturation of various inflammatory cells; others-such as II1r and Ccr4-are known to influence tumor angiogenesis and invasion. Hif1 $a$ was also significantly up-regulated, consistent with the rapid onset of tissue hypoxia observed upon Myc inhibition. Among the genes up-regulated following Myc inhibition were Il12b, ccl5, and Tlr2, all known to stimulate macrophage and NK activation.

Systemic expression of Omomyc in TRE-Omomyc; CMVrtTA;RIP1-Tag2 mice models a Myc inhibitory drug by blocking Myc function in both SV40 T/t-antigentransformed $\beta$ cells themselves and all other cell types in the RIP1-Tag2 tumors. Hence, it is not possible to determine the extent to which the tumoricidal effects of Myc inhibition are due to its effect on the tumor cells themselves versus the tumor microenvironment. To ascertain whether the dramatic collapse of the RIP1-Tag2 tumor microenvironment triggered by Myc inhibition is mediated through the tumor or the stromal compartments, we crossed our TRE-Omomyc;RIP1-Tag2 model

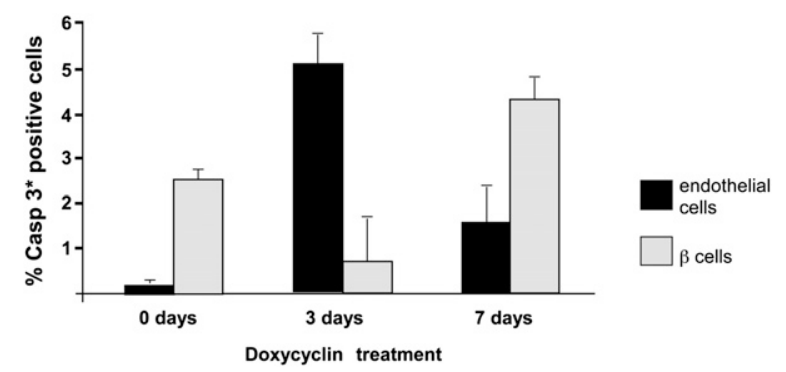

Figure 4. Relative kinetics of onset of endothelial versus $\beta$-cell apoptosis in RIP1-Tag2 tumors after Myc inhibition shows that vascular apoptosis precedes that of tumor cells. Quantification of active Caspase 3-positive (Casp $3^{\star}$ ) cells in the endothelial versus $\beta$-cell compartment of TRE-Omomyc;CMVrtTA;RIP1Tag2 tumors treated for 0,3 , or $7 \mathrm{~d}$ with doxycycline. Data are represented as mean $+\mathrm{SD}$. 

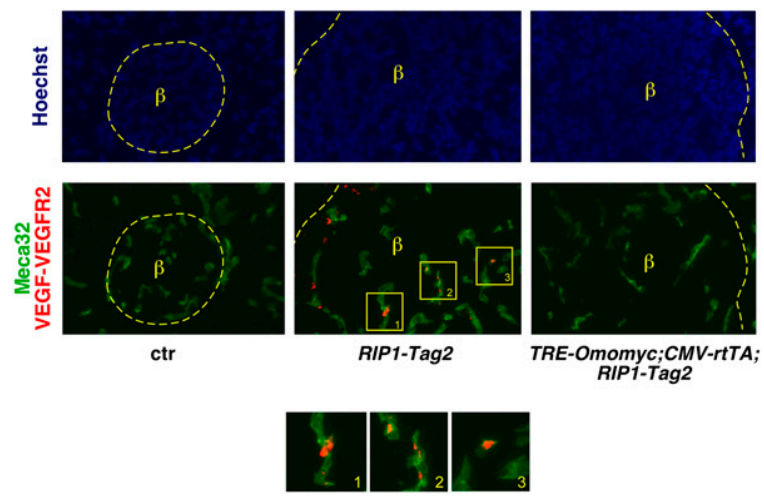

Figure 5. Endogenous Myc is required for binding of VEGFVEGFR interactions in RIP1-Tag2-induced tumors. Immunohistochemical analysis of VEGF:VEGF-R2 conjugates (in red) and Meca32 (in green) in sections of pancreata harvested from 14-wk-old nontransgenic control (ctr), RIP1-Tag2, and TREOmomyc;CMVrtTA;RIP1-Tag2 mice treated with doxycycline for 1 wk prior to sacrifice. Nuclear Hoechst counterstaining (in blue) of adjacent sections is shown in the top panels. Broken yellow lines mark tumor borders.

into the RIP-rtTA mouse so that doxycycline-inducible Omomyc is expressed exclusively in the $\beta$-cell compartment of each tumor. Inhibition of endogenous Myc only in the $\beta$-cell compartment of established RIP1-Tag2 tumors triggered rapid and widespread death of tumor endothelial cells (Fig. 8A) and tumor regression that was indistinguishable from that elicited by systemic Myc inhibition (Fig. 8B). Hence, maintenance of the vascular and inflammatory somatic microenvironment required to support SV40 T/t-antigen-driven RIP1-Tag2 tumors is absolutely dependent on endogenous Myc function within the tumor cell compartment.

One notional therapeutic advantage of targeting Myc function in cancers is its lack of functional redundancy, thus constraining opportunities for evolution of resistance. Since Myc inhibition is so effective at restraining RIP1-Tag2 tumor expansion, we reasoned that it must potently select for mechanisms by which tumor clones escape its grip, and, indeed, we did observe occasional individual islet tumors in TRE-Omomyc; CMVrtTA;RIP1Tag2 mice ( $\sim 0.4 \%$ of all tumors) that failed to regress following doxycycline treatment (Fig. 9A). To determine the mechanism of their apparent resistance, we immunohistochemically assayed these rogue tumors for expression of Omomyc. In every instance $(n=7)$, the nonresponsive tumors were devoid of detectable Omomyc expression (Fig. 9B), while surrounding exocrine tissues clearly retained doxycycline-inducible Omomyc immunoreactivity, as did all those tumors that regressed in response to doxycycline. Hence, the only mechanism we observe by which RIP1-Tag2 tumors acquire refractoriness to doxycycline is through loss of the Omomyc transgene. Of note, such Omomyc-negative tumors exhibited both VEGF:VEGFR2 ligate distribution and immune cell recruitment identical to that of control RIP1-Tag2 tumors (Fig. 9C,D). Taken together, these data support the notion that Myc is an essential and nonredundant target whose function is absolutely required for RIP1-Tag2 tumor maintenance.

\section{Discussion}

We previously used a systemic, switchable dominantnegative approach to show that lung tumors driven by oncogenic KRas in vivo are continuously dependent on endogenous Myc function for their maintenance at all stages of their evolution (Soucek et al. 2008). However, neither the mechanism by which Myc inhibition triggers tumor regression nor the general applicability of Myc inhibition to other tumor types driven by other oncogenic mechanisms was known. Here, we show that inhibition of endogenous Myc also triggers regression of pancreatic islet tumors driven by SV40 large $\mathrm{T}$ and small $\mathrm{t}$ antigens in RIP1-Tag2 mice. Just as with $\mathrm{KRas}^{G 12 D}$-driven lung tumors, Myc inhibition elicits rapid and widespread onset of tumor cell arrest and apoptosis, culminating in universal regression of all tumors, irrespective of tumor grade or stage. Hence, endogenous Myc function is an obligate requirement for RIP1-Tag2 tumor maintenance. Extensive studies have delineated the mechanisms by which SV40encoded large $\mathrm{T}$ antigen and small $\mathrm{t}$ antigen drive tumorigenesis. Large T antigen, SV40's principal oncoprotein, simultaneously incapacitates $\mathrm{Rb}$ and $\mathrm{p} 53$, the two principal tumor suppressors that restrain promiscuous proliferation. In parallel, small t modulates the pleiotropic impact of the PP2A Ser/Thr phosphatase that modulates multiple kinases downstream from Ras (Ahuja et al. 2005; Sablina and Hahn 2008). Any role played by endogenous Myc in SV40 transformation and tumorigenesis remains illdefined. At no stage of RIP1-Tag2 islet tumor evolution do we observe any overt up-regulation of c-Myc protein (Supplemental Fig. 7), suggesting that oncogenically activated Myc is never the oncogenic "driver" of these tumors, but, instead, is a downstream effector of the SV40 T/t oncoproteins. Indeed, $\mathrm{t}$ antigen has been shown to enhance

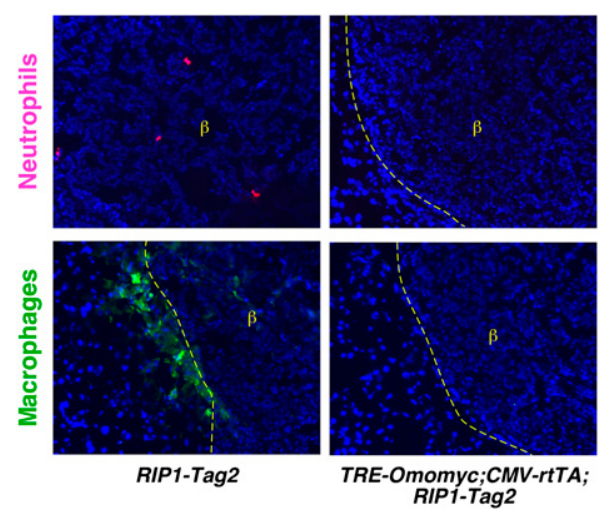

Figure 6. Endogenous Myc is required for retention of inflammatory cells by RIP1-Tag2 tumors. Immunohistochemical analysis of neutrophils (top panels, in pink over blue Hoechst counterstain) and macrophages (bottom panels, in green over blue Hoechst counterstain) in RIP1-Tag2 and TRE-Omomyc;CMVrtTA;RIP1Tag2 mice treated at $13 \mathrm{wk}$ of age for $3 \mathrm{~d}$ with doxycycline prior to sacrifice. Broken yellow lines delineate the islet borders. 


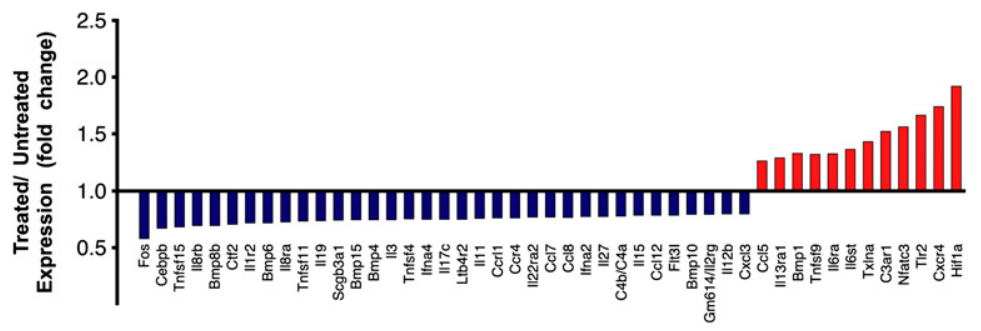

Figure 7. Myc inhibition rapidly modulates proinflammatory and anti-inflammatory genes. Comparative gene expression microarray analysis of cytokine, chemokine-encoding, and inflammation-related genes from laser-captured islets from 13-wk-old TRE-Omomyc; CMVrtTA;RIP1-Tag2 mice treated for $2 \mathrm{~d}$ with doxycycline versus untreated (sucrose only). The ratio of treated/untreated expression is represented by blue bars for down-regulated genes and red bars for up-regulated genes. Genes with treated/untreated expression ratios between 0.8 and 1.2 were excluded.
c-Myc activity by suppressing c-Myc dephosphorylation at Ser 62 and stabilizing the protein. Moreover, a stabilized c-Myc mutant can substitute for t-antigen expression in cell culture transformation assays (Sablina and Hahn 2008), again suggesting that endogenous Myc is a bona fide downstream effector of small $t$. Large T's association with p300 is also thought to promote c-Myc activity and potentiate large T-mediated cell cycle entry and cell transformation (Singhal et al. 2008). Of note, the $\beta$ cells within the regressed islet tumors in Omomyc-expressing TRE-Omomyc; CMVrtTA;RIP1-Tag2 mice remain clearly transformed even when Myc is inhibited. Hence, while growth and maintenance of macroscopic $\beta$-cell tumors driven by SV40 $\mathrm{T} / \mathrm{t}$ require endogenous $\mathrm{Myc}$, at least some cell-intrinsic aspects of SV40 $\mathrm{T} / \mathrm{t}$ cellular transformation do not. It is also intriguing that, despite their transformed cytological features, the regressed islet tumors in Omomyc-expressing RIP1-Tag2 mice exhibit size, distribution, and $\alpha$-cell to $\beta$-cell ratios similar to islets in normal littermates. Since the $\alpha$-cell compartment does not expand alongside that of $\beta$ cells during RIP1-Tag2 tumorigenesis, it would appear that the tumors regress back to both the size and site of their origins.

Tumor cells, like their normal counterparts, are completely dependent on their local microenvironment for provision of oxygen, nutrients, survival factors, and supporting stroma. Moreover, tumor growth, just like regeneration of normal tissues, requires a complex interplay between the expanding tissue and various accessory cellsmost notably inflammatory cells, endothelial cells, and fibroblasts. However, the reciprocal interaction between tumor cells and stroma is complicated because it evolves over time, making it difficult to address the most important therapeutic question: Which driving processes within a tumor are causally responsible for maintaining the tumor microenvironment, and do those processes originate in the tumor cells themselves or within the multifarious stromal compartment? While multiple studies indicate that ectopic Myc drives and maintains multiple aspects of the tumor microenvironment in transgenic Myc-driven tumors (Pelengaris et al. 1999, 2002; Shchors et al. 2006; Soucek et al. 2007), the role played by endogenous Myc in tumors driven by other oncogenic mechanisms in other tissue types has remained unclear. Our data unequivocally show that short-term systemic Myc inhibition in TREOmomyc;CMVrtTA;RIP1-Tag2 $\beta$-cell tumors is sufficient to trigger collapse of the tumor microenvironment, with concomitant death of endothelial cells, attenuation of inflammatory cells, vascular collapse, and hypoxia. Importantly, collapse of the tumor microenvironment temporally precedes detectable regression of tumors, implicating it as a potential cause, and certainly not just a consequence, of tumor regression. Moreover, since tumor regression occurs with identical kinetics and gross pathology when endogenous Myc is inhibited solely in the $\beta$-cell compartment, this confirms that it is endogenous

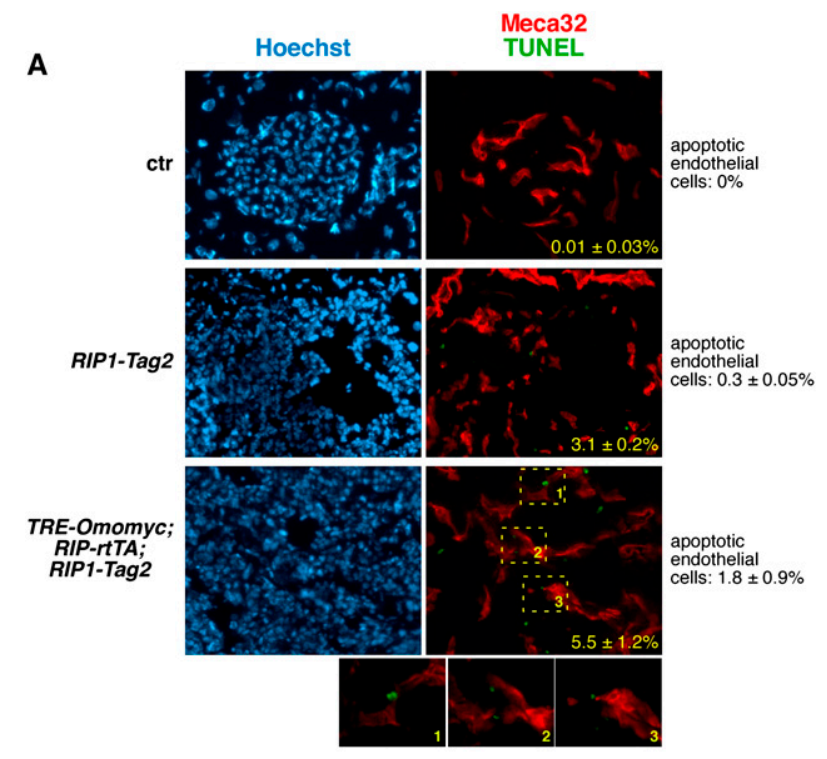

B

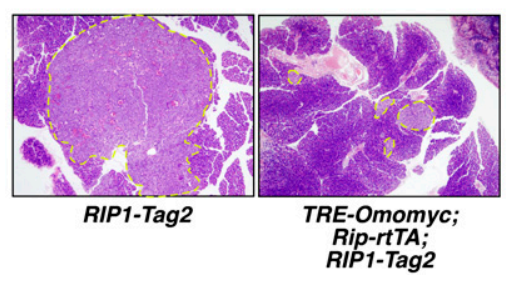

Figure 8. Myc inhibition only in the $\beta$-cell compartment triggers collapse of tumor vasculature and tumor regression. (A) Immunostaining for Meca32 (in red) and TUNEL staining (in green) in sections of pancreata harvested from 14-wk-old nontransgenic control (ctr), RIP1-Tag2, and TRE-Omomyc;RIP$r t T A ; R I P 1-T a g 2$ mice treated with doxycycline for $1 \mathrm{wk}$ prior to sacrifice. Insets $i-i i i$ show representative higher magnifications of TUNEL-positive apoptotic endothelial cells. $(B) \mathrm{H} \& \mathrm{E}$ staining of pancreata harvested from 14-wk-old RIP1-Tag2 and TREOmomyc;RIP-rtTA;RIP1-Tag2, the latter treated with doxycycline for $3 \mathrm{wk}$ prior to sacrifice. Yellow broken lines indicate the islet borders. Ages of mice at sacrifice are indicated in brackets. 
A

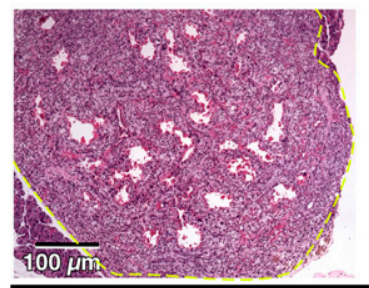

TRE-Omomyc;CMV-tTA;RIP1-Tag2

B

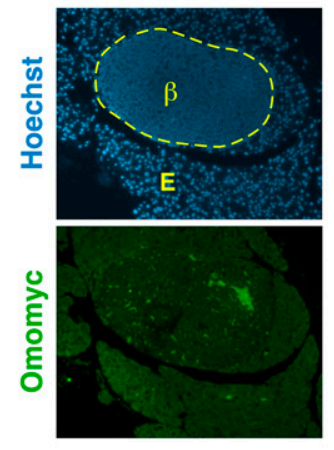

RIP1-Tag2

TRE-Omomyc;CMV-rtTA;RIP1-Tag2

C

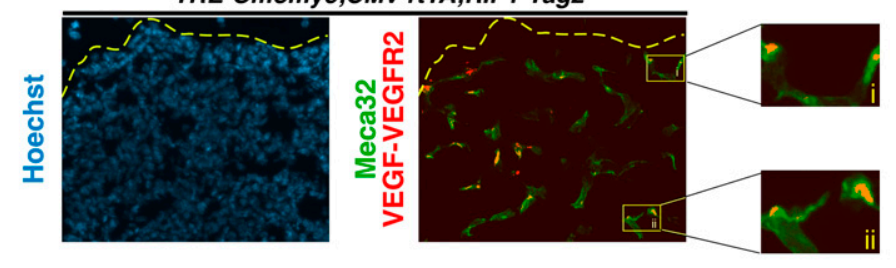

D

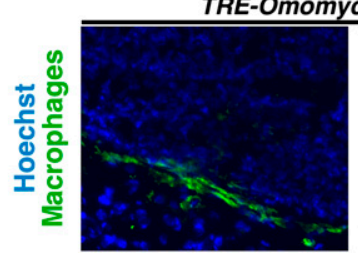

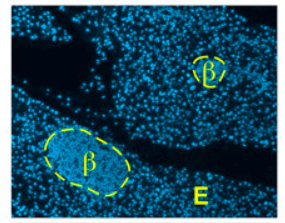

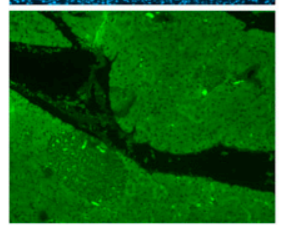
regressed
TRE-Omomyc;CMV-rtTA;RIP1-Tag2

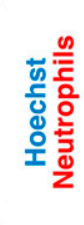

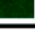
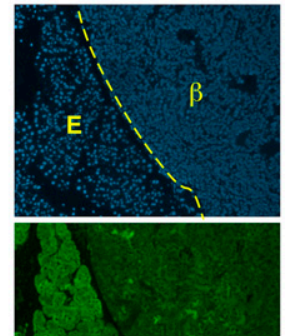

$x^{2}+x^{2}=0$ Insets show representative higher magnifications of VEGF:VEGF-R2-positive endothelial cells. $(D)$ Representative immunohistochemical staining for macrophages (left image, in green over blue Hoechst counterstain) and neutrophils (right image, in pink over blue Hoechst counterstain) of an islet tumor that failed to regress in a doxycycline-treated TRE-Omomyc;CMVrtTA;RIP1Tag2 mouse.
Myc within the tumor cells themselves that is responsible for instating and sustaining the signal cross-talk between tumor and microenvironment. Of course, we cannot exclude the possibility that endogenous Myc may also play a tumor maintenance role in cells within the stromal compartment, a role that could further augment the therapeutic impact of any systemic Myc inhibitory therapy. Of note, our data support the notion that targeting Myc, a nonredundant obligate effector lying upstream of the plethora of signals that maintain the tumor microenvironment, may sidestep the adaptive compensation that bedevils existing anti-angiogenic and anti-inflammatory cancer therapies. These same obligate and nonredundant attributes also have the potential to minimize the other principal reason for failure of targeted therapies in cancer treatment; namely, the evolution within tumors of de novo mechanisms that circumvent the tumor cells' initial requirement for that target. In this regard, it is interesting that, out of several hundred individual $\beta$-cell tumors, each comprised of many thousands of tumor cells in which both $\mathrm{Rb}$ and $\mathrm{p} 53$ had been concomitantly in- capacitated, the only occasional tumors in TRE-Omomyc; CMVrtTA;RIP1-Tag2 mice that were refractory to doxycycline had all lost expression of the Omomyc transgene, consistent with the idea that Myc inhibition per se cannot be circumvented by compensatory or evolutionary mechanisms. This, together with the remarkable mildness of side effects that systemic Myc inhibition elicits (Soucek et al. 2008), reinforces the notion that inhibiting Myc is an exceptionally promising and generic strategy for treating diverse cancers.

\section{Materials and methods}

\section{Generation and maintenance of genetically engineered mice}

TRE-Omomyc and RIP1-Tag2 mice have been described previously (Hanahan 1985; Soucek et al. 2008). CMVrtTA $[\mathrm{Tg}(\mathrm{rtTAhCMV}) 4 \mathrm{Bj} / \mathrm{T}]$ mice were purchased from the Jackson Laboratory. RIP-rtTA [Tg(Ins2-rtTA)2Efr/J] mice were obtained from Dr. Shimon Efrat. Omomyc expression was systemically induced in TRE-Omomyc;CMVrtTA mice by addition of doxycycline $(2 \mathrm{mg} / \mathrm{mL}$, plus $5 \%$ sucrose) to their drinking water. 
Sucrose only was administered to control mice. Animals were maintained and treated in accordance with protocols approved by the Institutional Animal Care and Use Committee (IACUC) at the University of California at San Francisco (UCSF). For each experiment, cohorts of at least five mice were used for each data point.

\section{Tissue preparation and histology}

Mice were euthanized and cardiac-perfused with PBS followed by zinc-buffered formalin. Pancreata were removed, fixed overnight in zinc-buffered formalin, and processed for paraffin embedding. Tissue sections $(5 \mu \mathrm{m})$ were stained with hematoxylin and eosin (H\&E) using standard reagents and protocols. For frozen sections, pancreata were embedded in OCT, frozen on dry ice, and stored at $-80^{\circ} \mathrm{C}$.

\section{Immunohistochemistry}

For immunohistochemical analysis, sections were deparaffinized, rehydrated, and subjected to high-temperature antigen retrieval in $10 \mathrm{mM}$ citrate buffer $(\mathrm{pH}$ 6.0). Primary antibodies were as follows: rabbit monoclonal anti-Ki67 (clone SP6) (Lab Vision), rabbit polyclonal anti-Omomyc (prepared in-house, Evan laboratory) (Soucek et al. 2004), rat monoclonal anti-MECA32 (BD PharMingen), rabbit polyclonal anti-cleaved Caspase-3 (R\&D Systems), mouse monoclonal anti-VEGF:VEGFR complex (clone Gv39M; EastCoast Bio), guinea pig polyclonal anti-insulin (Millipore), rabbit anti-Tag (prepared in the Hanahan laboratory) (Casanovas et al. 2005), rat monoclonal anti-macrophage F4/80 antigen (AbD Serotec), rat monoclonal anti-neutrophils (Clone 7/4; Cedarlane), and rabbit polyclonal anti-NG2 (Millipore). Primary antibodies were applied for $2 \mathrm{~h}$ in blocking buffer ( $2.5 \%$ BSA, $5 \%$ goat serum, $0.3 \%$ Triton $\mathrm{X}-100$ in PBS), sections were washed, and species-appropriate secondary Alexa Fluor 488 dye-conjugated antibodies (Amersham) or Vectastain ABC kit and DAB reagents (Vector Laboratories) were applied. Fluorescence antibody-labeled slides were mounted in DAKO fluorescent mounting medium containing $1 \mu \mathrm{g} / \mathrm{mL}$ Hoechst counterstain. HRP-conjugated secondary antibodies were visualized by DAB staining (Vector Laboratories). Apoptotic cells were detected by TUNEL (ApopTag Detection kit; Chemicon International) or by expression of activated caspase 3 . To stain tissues systemically for hypoxia, $60 \mathrm{mg} / \mathrm{kg}$ hypoxyprobe-1 (1-\{[2-hydroxy-3-piperdinyl] propyl\}-2-nitroimidazole hydrochloride) was administered intraperitoneally in saline $15 \mathrm{~min}$ prior to euthanasia. Protein adducts of reductively activated pimonidazole were identified immunohistochemically in fixed tissues with a monoclonal antibody against hypoxyprobe-1. Images were collected with an Axiovert 5100 TV inverted fluorescence microscope (Zeiss) and Open Lab 3.5.1 software, or with an Axiovert 100 inverted microscope (Zeiss) equipped with a Hamamatsu Orca digital camera.

\section{Microarray analysis}

For expression profiling, 13-wk-old TRE-Omomyc;CMVrtTA; RIP1-Tag2 mice were treated with either doxycyline $(2 \mathrm{mg} / \mathrm{mL}$, plus $5 \%$ sucrose; referred to as "treated") or $5 \%$ sucrose (referred to as "untreated") in their drinking water for $2 \mathrm{~d}$. Pancreata were isolated and embedded immediately with OCT. Fresh-frozen pancreatic sections were fixed in ice-cold $70 \%$ ethanol before laser-capture microdissection. A modified H\&E staining protocol was used for microscopic visualization of islets while preserving RNA integrity (Goldsworthy et al. 1999; Luzzi et al. 2001). Tumor islets were isolated with a Zeiss PALM MicroBeam Laser Microdissection system. For each independent sample $(n=6$ : three treated and three untreated), three to six tumors were captured from four to six adjacent tissue sections and pooled for RNA isolation. Total RNA was isolated using an Arcturus PicoPure RNA isolation kit, and RNA was amplified with a NuGEN FFPE kit (NuGEN Technologies, Inc.) and processed for hybridization on Affymetrix mouse set ST1.0. Robust multiarray (RMA) analysis using Affymetrix Power Tool "APT" software was used to normalize and summarize probe level intensities, and any transcript clusters that, after normalization, had a logbase- 2 score $<3.0$ were discarded. The fold change in expression for each gene was calculated from the ratio of median expression of treated samples versus untreated.

\section{Acknowledgments}

We are indebted to Fanya Rostker for her technical assistance, and to our colleagues in the Evan laboratory for their invaluable criticism and advice. We thank Christopher Chiu for technical advice, and Lisa Coussens' laboratory for reagents. This work was supported by grants from the National Cancer Institute (2R01 CA98018), the Susan G. Komen Breast Cancer Foundation, the Samuel R. Waxman Cancer Research Foundation, the Association for International Cancer Research, and the Bear Necessities Pediatric Cancer Foundation.

\section{References}

Ahuja D, Saenz-Robles MT, Pipas JM. 2005. SV40 large T antigen targets multiple cellular pathways to elicit cellular transformation. Oncogene 24: 7729-7745.

Baskar JF, Smith PP, Nilaver G, Jupp RA, Hoffmann S, Peffer NJ, Tenney DJ, Colberg-Poley AM, Ghazal P, Nelson JA. 1996. The enhancer domain of the human cytomegalovirus major immediate-early promoter determines cell type-specific expression in transgenic mice. J Virol 70: 3207-3214.

Bergers G, Hanahan D. 2008. Modes of resistance to antiangiogenic therapy. Nat Rev Cancer 8: 592-603.

Bergers G, Brekken R, McMahon G, Vu TH, Itoh T, Tamaki K, Tanzawa K, Thorpe P, Itohara S, Werb Z, et al. 2000. Matrix metalloproteinase- 9 triggers the angiogenic switch during carcinogenesis. Nat Cell Biol 2: 737-744.

Brekken RA, Huang X, King SW, Thorpe PE. 1998. Vascular endothelial growth factor as a marker of tumor endothelium. Cancer Res 58: 1952-1959.

Casanovas O, Hicklin DJ, Bergers G, Hanahan D. 2005. Drug resistance by evasion of antiangiogenic targeting of VEGF signaling in late-stage pancreatic islet tumors. Cancer Cell 8: 299-309.

Christofori G, Hanahan D. 1994. Molecular dissection of multistage tumorigenesis in transgenic mice. Semin Cancer Biol 5: 3-12.

Coussens LM, Fingleton B, Matrisian LM. 2002. Matrix metalloproteinase inhibitors and cancer: trials and tribulations. Science 295: 2387-2392.

Fingleton B. 2003. Matrix metalloproteinase inhibitors for cancer therapy: the current situation and future prospects. Expert Opin Ther Targets 7: 385-397.

Folkman J, Watson K, Ingber D, Hanahan D. 1989. Induction of angiogenesis during the transition from hyperplasia to neoplasia. Nature 339: 58-61.

Furth PA, Hennighausen L, Baker C, Beatty B, Woychick R. 1991. The variability in activity of the universally expressed human cytomegalovirus immediate early gene 1 enhancer/ promoter in transgenic mice. Nucleic Acids Res 19: 62056208. 
Sodir et al.

Goldsworthy SM, Stockton PS, Trempus CS, Foley JF, Maronpot RR. 1999. Effects of fixation on RNA extraction and amplification from laser capture microdissected tissue. Mol Carcinog 25: 86-91.

Hanahan D. 1985. Heritable formation of pancreatic $\beta$-cell tumours in transgenic mice expressing recombinant insulin/ simian virus 40 oncogenes. Nature 315: 115-122.

Kothary R, Barton SC, Franz T, Norris ML, Hettle S, Surani MA. 1991. Unusual cell specific expression of a major human cytomegalovirus immediate early gene promoter-lacZ hybrid gene in transgenic mouse embryos. Mech Dev 35: 25-31.

Luzzi V, Holtschlag V, Watson MA. 2001. Expression profiling of ductal carcinoma in situ by laser capture microdissection and high-density oligonucleotide arrays. Am I Pathol 158: 2005-2010.

Meyer N, Penn LZ. 2008. Reflecting on 25 years with MYC. Nat Rev Cancer 8: 976-990.

Miller KD, Sweeney CJ, Sledge GW Jr. 2005. Can tumor angiogenesis be inhibited without resistance? EXS 2005: 95-112.

Nozawa H, Chiu C, Hanahan D. 2006. Infiltrating neutrophils mediate the initial angiogenic switch in a mouse model of multistage carcinogenesis. Proc Natl Acad Sci 103: 1249312498.

Pelengaris S, Littlewood T, Khan M, Elia G, Evan G. 1999. Reversible activation of c-Myc in skin: induction of a complex neoplastic phenotype by a single oncogenic lesion. Mol Cell 3: 565-577.

Pelengaris S, Khan M, Evan GI. 2002. Suppression of Mycinduced apoptosis in $\beta$ cells exposes multiple oncogenic properties of Myc and triggers carcinogenic progression. Cell 109: 321-334.

Sablina AA, Hahn W. 2008. SV40 small T antigen and PP2A phosphatase in cell transformation. Cancer Metastasis ReV 27: 137-146.

Shchors K, Shchors E, Rostker F, Lawlor ER, Brown-Swigart L, Evan GI. 2006. The Myc-dependent angiogenic switch in tumors is mediated by interleukin $1 \beta$. Genes Dev 20: $2527-$ 2538.

Shojaei F, Singh M, Thompson JD, Ferrara N. 2008. Role of Bv8 in neutrophil-dependent angiogenesis in a transgenic model of cancer progression. Proc Natl Acad Sci 105: 2640-2645.

Singhal G, Kadeppagari RK, Sankar N, Thimmapaya B. 2008. Simian virus 40 large T overcomes p300 repression of c-Myc. Virology 377: 227-232.

Soucek L, Helmer-Citterich M, Sacco A, Jucker R, Cesareni G, Nasi S. 1998. Design and properties of a Myc derivative that efficiently homodimerizes. Oncogene 17: 2463-2472.

Soucek L, Jucker R, Panacchia L, Ricordy R, Tato F, Nasi S. 2002. Omomyc, a potential Myc dominant negative, enhances Myc-induced apoptosis. Cancer Res 62: 3507-3510.

Soucek L, Nasi S, Evan GI. 2004. Omomyc expression in skin prevents Myc-induced papillomatosis. Cell Death Differ 11: 1038-1045.

Soucek L, Lawlor E, Soto D, Shchors K, Swigart L, Evan G. 2007. Mast cells are required for angiogenesis and macroscopic expansion of Myc-induced pancreatic islet tumors. Nat Med 13: $1211-1218$.

Soucek L, Whitfield J, Martins CP, Finch AJ, Murphy DJ, Sodir NM, Karnezis AN, Swigart LB, Nasi S, Evan GI. 2008. Modelling Myc inhibition as a cancer therapy. Nature 455: 679-683. 


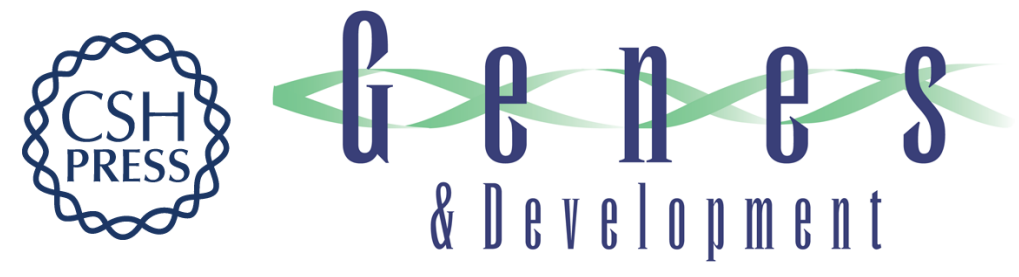

\section{Endogenous Myc maintains the tumor microenvironment}

Nicole M. Sodir, Lamorna Brown Swigart, Anthony N. Karnezis, et al.

Genes Dev. 2011, 25: originally published online April 8, 2011

Access the most recent version at doi:10.1101/gad.2038411

\footnotetext{
Supplemental

Material

http://genesdev.cshlp.org/content/suppl/2011/04/04/gad.2038411.DC1

Related Content

Addicted to Mycbut why?

Björn von Eyss and Martin Eilers

Genes Dev. May , 2011 25: 895-897

References This article cites 29 articles, 7 of which can be accessed free at: http://genesdev.cshlp.org/content/25/9/907.full.html\#ref-list-1

Articles cited in:

http://genesdev.cshlp.org/content/25/9/907.full.html\#related-urls

License

Email Alerting

Service

Receive free email alerts when new articles cite this article - sign up in the box at the top right corner of the article or click here.
}

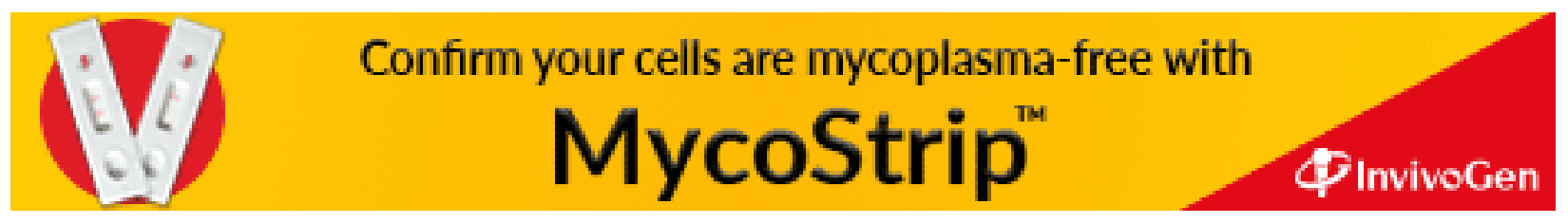

